\title{
An Effective Method of Accelerating Bose Gases Using Magnetic Coils
}

\author{
Haichang Lu（卢海昌）, Yueyang Zhai(翟跃阳), Ruizhi Pan(潘睿智), and Shifeng Yang (杨仕锋)* \\ School of Electronics Engineering and Computer Science, \\ Peking University, Beijing 100871, People's Republic of China
}

(Dated: February 23, 2014)

\begin{abstract}
Based on the experimental device which is a non-uniform magnetic field to trap an atom, we show how to obtain a certain velocity of a Bose gas by controlling the magnetic coils. By comparing the relationship of different current supply and delay time versus the ultimate velocity of the atom,we theoretically predict the method of accelerating the gases to an expected velocity. This method is of great convenience and significance for the applications in cold atom physics and precision measurements.
\end{abstract}

Keywords: Magnetic Control, Bose-Einstein Condensation

PACS numbers: 37.10.Gh, 37.10.De

The realization of Bose-Einstein condensation (BEC) has rendered studies of quantum phenomena at ultra-cold temperature possible. It has become a very powerful tool in the fundamental research such as atomic interferometry [1], Bragg diffraction [2], atomic clock [3, 4] and quantum phase transition [5]. For all these research areas, manipulating the external state of a cold atom, i.e. its momentum, is a key technique. So far, most of the experiments studies have been implemented by accelerating atoms. In Ref.[6], Bragg diffraction that indicates the long-range coherence of BEC was studied by using a moving atom cloud. The condensate was accelerated and the transition from the pure super-radiant regime to the Bragg scattering regime appeared. Bloch oscillations of Bose-Einstein condensates with a certain velocity in one dimensional optical lattice was observed in Ref.[7]. However, the average velocity of the BEC was derived from the occupation numbers of the different momentum states after a flight time, which was not directly seen from the imaging picture.

There are several methods driving neutral atoms to move. The usual way is using a pair of counterpropagating Raman laser beams with a proper frequency difference to illuminate atoms. The energy generated from the light frequency difference is absorbed and then transferred to the atom's kinetic energy. For driving atoms, complicated optical accessories are applied to shift the light frequency and an additional light path is needed. With two designed light pulses that also produced by two counter-propagating light beams with the same frequency, a BEC cloud can be split into several momentum parts. However, a pure momentum is impossible to achieve by using this kind of pulses and strong light power is necessary [8]. Here we present an approach to accelerate the cold atoms to a certain velocity by utilizing

\footnotetext{
*Electronic address: yangshifeng111@126.com
}

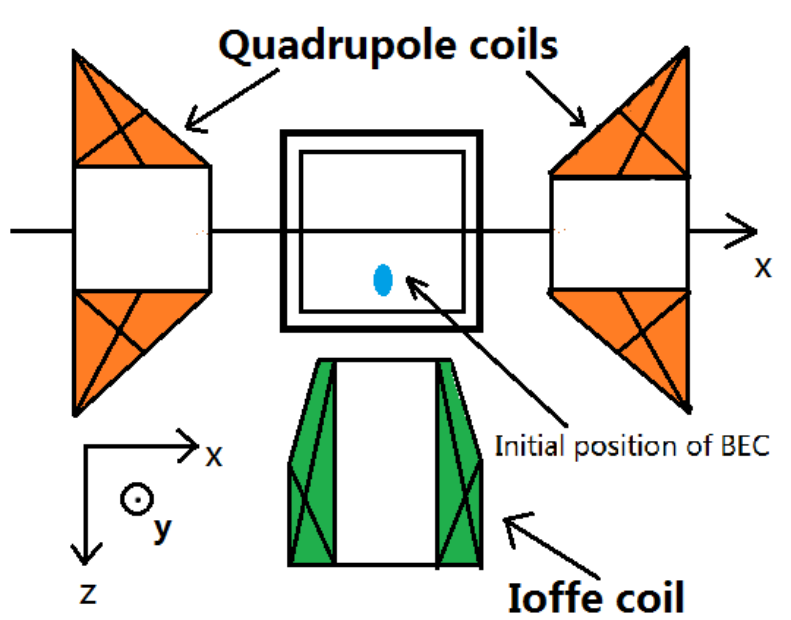

FIG. 1: The structure of QUIC trap. The coils in orange is quadruople coil,which consists of two coils with the same structure but inverse current (Anti-Helmholtz coils), while the coil in green is called Ioffe coil. The blue dot shows the initial position of BEC which is also the bottom of the static QUIC $\operatorname{trap}\left(z_{0}=8.025 \mathrm{~mm}\right)$.

a dynamic magnetic field. First we elaborate the interactions between neutral atom and magnetic field. Then by adjusting the parameters of the magnetic system, we can control the acceleration of the atom to an expected velocity. Our theoretical and experimental results consist well. Finally we give the maximal values of atom velocity with different conditions under this method in theory.

The common procedure to generate a BEC is transforming atoms that are in magnetic-optical traps(MOT) into magnetic trap.After loading the cold atoms into the magnetic trap, evaporation cooling is used to remove the high kinetic energy atoms. About one-thousandth of atoms can be kept and finally becoming the BEC. In our experiment, we use quadrupole coils(Anti-Helmholtz 
coils) and Ioffe coil[9] to constitute a quadrupole-Ioffeconfiguration(QUIC)trap[10](FIG. 1). All the coils are settled around an ultra-vacuum glass container on a copper supporter that can be cooled by water. To prevent the eddy current in the copper frame when being energized, some crucial parts in the holder are fabricated with gaps.

The potential energy of atoms in QUIC trap $U(r, z)$ can be written as

$$
U(r, z)=U_{0}+\frac{1}{2} m \omega_{r}^{2} r^{2}+\frac{1}{2} m \omega_{z}^{2} z^{2}
$$

where $\mathrm{r}$ is the radial direction, $\mathrm{z}$ is alone axial of the Ioffe coil, $\omega_{r}$ and $\omega_{z}$ are radial frequency and axial frequency in QUIC trap, respectively. The BEC in such a gradient magnetic field will be stressed by a force

$$
\vec{F}=-\nabla(-\mu \cdot \vec{B})=-\nabla\left(m_{F} g_{F} \mu_{B} \vec{B}\right)=-\nabla\left(\mu_{B} \vec{B}\right)
$$

for an atom in $\mid F=2, m_{F}=2>$ state, where $\mu$ is the atomic magnetic moment and $\vec{B}$ is the magnetic intensity in the trapping area. When turning off the current in coils, the current is not perfectly decreased to zero immediately but with a decay time. In principle, we can choose different decay times of the currents in Ioffe and quadrupole coil to get certain magnetic field that can accelerate atoms by gradient force $\vec{F}$. If the current in Ioffe coil decays faster than in quadrupole coil, then the atoms will move in a region where quadrupole magnetic field has the major effect. In that case, atoms should be projected towards to the center of the quadrupole trap.

With the force at direction $z$ alone the axial of Ioffe coil[13]

$$
\begin{aligned}
F(z, t) & =m \ddot{z}(t)=-\mu_{B} \frac{\partial B(z(t), t)}{\partial z} \\
& =-\mu_{B}\left[I_{i} \frac{\partial}{\partial z} f_{i}(z) e^{-t / \tau_{i}}+I_{q} \frac{\partial}{\partial z} f_{q}(z) e^{-t / \tau_{q}}\right]
\end{aligned}
$$

we can get the expected atom velocity, where $m$ is the atomic mass, $f_{i}\left(f_{q}\right), I_{i}\left(I_{q}\right)$, and $\tau_{i}\left(\tau_{q}\right)$ are respectively the structure function, driven currents, and decay time of the Ioffe-type (quadrupole) trap [14]. We achieved it by optimizing the discharging loop of current control circuit. When shutting down the power supply system, the oscilloscope detected that the quadrupole current $I_{q}$ and Ioffe current $I_{i}$ attenuate in a pattern like exponent . The characteristic time of $I_{i}$ is approximately $66 \mu s$ while $I_{q}$ is $87 \mu \mathrm{s}$. In the whole process, there are magnetic zero points, which will cause the abscondence of an atom if approaching the points(Majorana transition). What we can assure is that the response of an atom to the external field is so retardant that most of the atoms are far from the bottom of the trap. With our calculations, when the current in both Ioffe coil and quadrupole coil is $21 \mathrm{~A}\left(I_{i}=I_{q}=21 \mathrm{~A}\right)$, an atomic velocity of $1.7 \mathrm{~mm} / \mathrm{s}$ (corresponds to $0.5 \hbar k$ ) can be achieved.

To make the trap work steadily, we need a stabilized power supply or constant current source controlled by

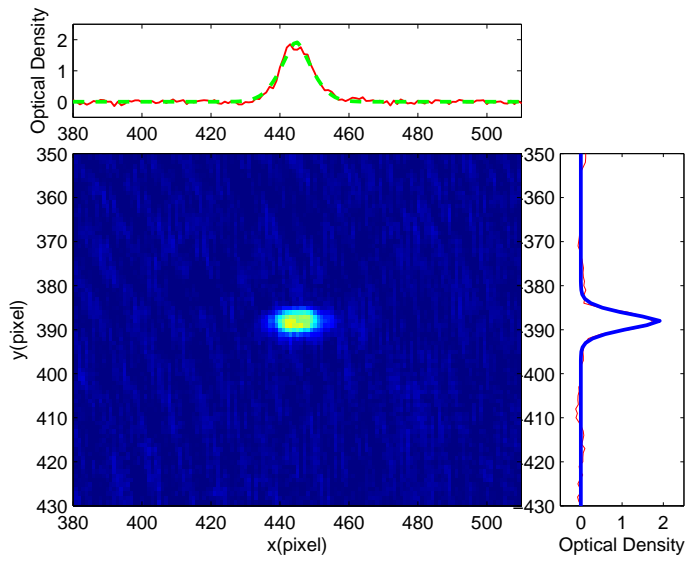

FIG. 2: The atomic number distribution in two dimension after $1.8 \mathrm{~ms}$, describing by optical density. The subplot in the figure collects the data from the digital picture(red line) and fits into the anti-parabola distribution(green dash line and blue line) which indicate the Bose-Einstein Condensate. (1 $\operatorname{pixel} \approx 6.8 \mu \mathrm{m})$.

digital or analogue signals. One of the advantages of QUIC trap is that the current normally required is merely $21 \mathrm{~A}$, so the power dissipation is merely $520 \mathrm{~W}$, provided that the impedances of quadrupole coil and Ioffe coil are $0.7 \Omega$ and $0.2 \Omega$, respectively. Using QUIC trap apparently makes the control and cooling much easier compared to using other traps. The power source is TAKASAGO' $\mathrm{s}$ GP $35-50 R$. It has two operating modes: constant voltage mode in which the stability can reach $10^{-4}$, and constant current mode in which the stability can reach $10^{-3}$. We chose constant voltage mode, and adopted selfmade circuits to control the current in both quadrupole coil and Ioffe coil. By applying the negative feedback system, the stability of the circuit can reach $10^{-5}$.

In our experiment, we prepared a cigar-shaped BEC sample of $10^{5}{ }^{87} R b$ atoms in the quadrupole-Ioffe configuration trap, of which the axial frequency is $20 \mathrm{~Hz}$, the radial frequency is $220 \mathrm{~Hz}$ and the quantum state is $\mid F=2, m_{F}=2>$. Before releasing, the atoms stay still in the bottom of the trap. Then we shut down the current of the QUIC trap that leads a horizontal projectile motion of BEC. In order to detect the location of atom$\mathrm{s}$, after switching off the magnetic trap, we imaged the expanding cloud at releasing time $\Delta t$ by using resonant probe light propagating along the $x$ axis. The CCD camera [15]recorded a slight movement from left to right for approximately $3.67 \mu \mathrm{m}$ within $1.8 \mathrm{~ms}$, as well as an anti-parabola distribution of the condensate with the radial length vibrating periodically(FIG. 2). We study the atomic velocity from the center of the distribution movement within a certain time period $\Delta t$. After measuring the locations of the atom at different time, we can detect from (FIG. 3) that the atom, regardless of its acceleration which is not prominent in early time, travels in a 


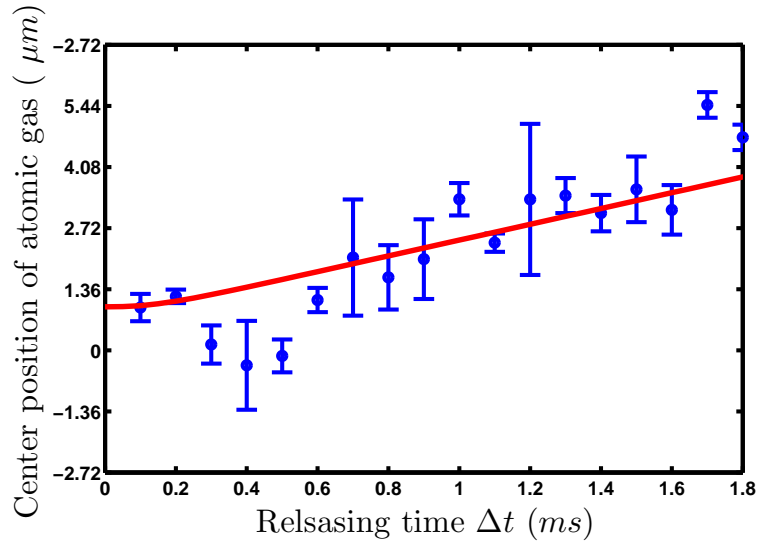

FIG. 3: The movement of atomic gas via different releasing time $\Delta t$. The blue dots show the experimental results, and each dot indicates the mean value of the data collected from the three repeated experiments. The red line is the theoretical curve with our experimental parameters as inputs, where $I_{i}=$ $I_{q}=21 A, \tau_{i}=66 \mu s$ and $\tau_{q}=87 \mu s$.

constant velocity of $1.7 \mathrm{~mm} / \mathrm{s}$.

By using this method of accelerating cold atoms, we are able to generate high enough atomic velocity theoretically.There are several factors influencing how atoms run in the trap, which consequently contribute to the condition of the bottom and its vicinity,thus determine how an independent(without interaction) atom responses to the change of external field. We compared the two eminent factors, the current supply and the discrepancy of characteristic times of two parts of the coils.

Firstly, we study the difference of coils' current in both Ioffe coil and quadrupole coil with a fixed decay times

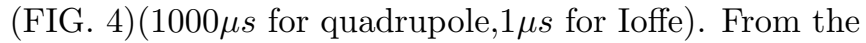
picture we can sketchily see that the initial behaviors of the velocity are mutually different and the final velocity of the atom depends linearly on the current supply. The current only change the shape of the bottom linearly, derived from the linear relationship of current and magnetic intensity. However, it is hasty to conclude the linear relationship of the final velocity and current for the reactions of an atom are not linear. Fortunately, we already know that an atom like Rubidium responses so sluggish that in the whole process it is far from catching up with the movement of the bottom. With this approximation and the fugacity of the field, by judging the initial effect posing to the atom, the final velocities linearly depend on the current supply. With this condition at a maximal current value of $30 A$ a velocity of $35 \hbar k$ can be achieved.

Then we calculate another situation. The current in both Ioffe coil and quadrupole coil is $30 \mathrm{~A}$ with $\tau_{i}=1 \mu \mathrm{s}$. By changing the characteristic time of quadrupole $\tau_{q}$, we change the speed of the bottom of the trap. The initial accelerations are identical for the four curves, which means this factor don't affect the conduct at the beginning of shutting down the power due to the fact that

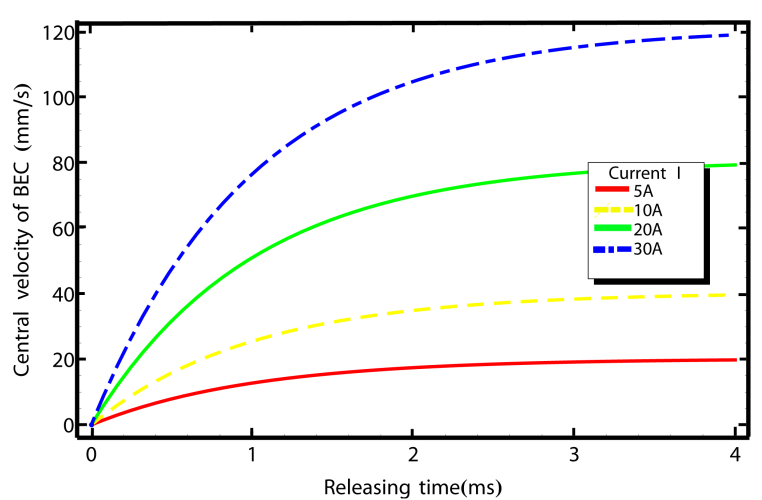

FIG. 4: The relations of velocity and time with different initial currents and with same characteristic time of Ioffe coil, $1 \mu s$, of quadrupole coil, $1000 \mu \mathrm{s}$.

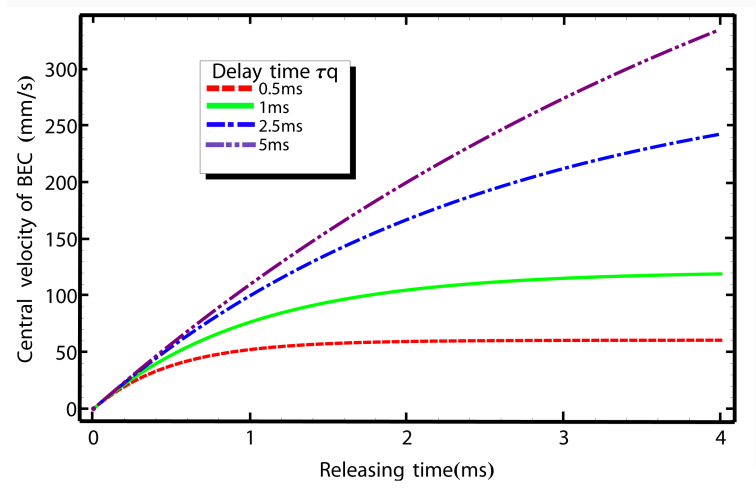

FIG. 5: The relations of velocity and time sharing same initial current $30 \mathrm{~A}$ and the characteristic time of Ioffe coil $1 \mu \mathrm{s}$, but with different characteristic time of quadrupole.

it does not change the intensity but the position. Subsequently, this non-linear factor results in disparate accelerations at the point of the atom experience and the times the velocities become asymptotically flat. Unlike the first factor whose fives velocities almost end up a constant simultaneously, a larger discrepancy of the two characteristic times will exert a longer effect to the atom. The red curve $\left(\tau_{q}=0.5 \mathrm{~ms}\right)$ (FIG. 5)only experiences a millisecond reaching only $15 \hbar k$, whereas the purple $\operatorname{curve}\left(\tau_{q}=5 \mathrm{~ms}\right)$ has a much larger velocity of $97 \hbar k$ within $4 \mathrm{~ms}$. Usually, it is more convenient to take the advantage of this factor to accelerate atomic gases to an expected velocity. However, we should carefully choose parameters of the current delay time in order to make the accelerating duration time within a certain valid time. As in the gravitational field, after $5 \mathrm{~ms}$ the atoms will drop $123 \mu \mathrm{m}$ from the center of magnetic trap, this calculation model would no longer simulate the real condition.

In summary, we have both experimentally and theoretically studied the behavior of cold atoms in magnetic trap. As QUIC trap is a common trap generating BEC, it is very convenient to use this method without adding or changing coil. With current control electric circuit 
we can generate a low-noise rapid-response quadrupoleIoffe configuration trap. By using different designed current delay time, that leads to a gradient magnetic field, atoms can be accelerated to an expected velocity. According to our calculations, an atomic velocity of $97 \hbar k$ can be achieved. In the subsequent experiment, we accelerated atomic gases within $400 \mathrm{~ms}$ to reach to a veloc- ity of $0.32 \hbar k$ and observed a magneto-optical diffraction of ultra-cold atom[16]. In that case, this method can be taken as an effective and convenient tool for moving BEC into the QUIC trap.

We thank X.J.Zhou for helpful discussion. This work is supported by the National Fundamental Research Program of China under Grant No. 2011CB921501.
[1] Cronin A D, Schmiedmayer J and Pritchard D E 2009 Rev. Mod. Phys. 81 1051;

[2] Kozuma M, Deng L, Hagley W, Wen J, Lutwak R, Helmerson K, Rolston S L and Phillips W D 1999 Phys. Rev. Lett. 82, 871;

[3] Takamoto M, Hong F L, Higashi R and Katori H 2005 Nature 435 321;

[4] Zheng Y N, Zhou X J, Chen J B and Chen X Z 2006 Chin. Phys. Lett. 7 1687;

[5] Greiner M, Mandel O, Esslinger T, Hänsch T W and Bloch I 2002 Nature 415 39;

[6] Fallani L, Fort C, Piovella N, Cola M, Cataliotti F S, Inguscio M and Bonifacio R 2005 Phys. Rev. A 71 033612;

[7] Morsch O, Müller J H, Cristiani M, Ciampini D and Arimondo E 2001 Phys. Rev. Lett. 87 140402;

[8] Xiong W, Yue X G, Wang Z K, Zhou X J, and Chen X Z 2011 Phys. Rev. A 84 043616;

[9] Pritchard D E, 1983 Phys. Rev. Lett. 51 1336;
[10] Esslinger T, Bloch I, and Hänsch T. W. 1998 Phys. Rev. A 58 R2664;

[11] Chiow S, Kovachy T, Chien H, and Kasevich M A 2011 Phys. Rev. Lett. 107 130403;

[12] Han J S, Xu X P, Zhang H C and Wang Y Z Chin.Phys.B Vol.22,No.2(2013) 023702

[13] Reichle R, Leibfried D, Blakestad R B, Britton J, Jost J D, Knill E, Langer C, Ozeri R, Seidelin S and Wineland D J 2006 Fortschr. Phys. 54666

[14] Xia L, Xu X, Guo R,Yang F, Xiong W, Li J T, Ma Q L, Zhou X J, Guo H, and Chen X Z 2008 Phys. Rev. A 77, 043622.

[15] Xia T, Zhou S Y, Chen P, Li L, Hong T and Wang Y Z Chin.Phys.Lett. Vol.27,No.2(2010)023701

[16] Zhai Y Y, Zhang P, Chen X Z,Dong G J and Zhou X J 2013 Phys.Rev A 88, 053629. 